PROCEEDINGS OF THE

AMERICAN MATHEMATICAL SOCIETY

Volume 132, Number 6, Pages 1797-1804

S 0002-9939(03)07377-5

Article electronically published on December 31, 2003

\title{
A CHARACTERIZATION OF THE LAGRANGIAN PSEUDOSPHERE
}

\author{
ILDEFONSO CASTRO AND FRANCISCO URBANO \\ (Communicated by Jon G. Wolfson) \\ Dedicated to B. Y. Chen on his 60th birthday
}

\begin{abstract}
We characterize the Lagrangian pseudosphere as the only branched Lagrangian immersion of a sphere in complex Euclidean plane with constant length mean curvature vector.
\end{abstract}

\section{Statement of the Result}

Let $\Omega$ be the Kähler two-form on the complex Euclidean plane $\mathbb{C}^{2}$ given by $\Omega(v, w)=\langle v, J w\rangle$, with $\langle$,$\rangle the Euclidean metric and J$ the complex structure on $\mathbb{C}^{2}$. An immersion $\phi$ of an oriented surface $\Sigma$ in $\mathbb{C}^{2}$ is called Lagrangian if $\phi^{*} \Omega \equiv 0$. It is well known that if $\sigma$ is the second fundamental form of $\phi$, then the 3-tensor $\langle\sigma(-,-), J-\rangle$ on $\Sigma$ is totally symmetric. From here it follows that if $H$ denotes the mean curvature vector of $\phi$, then the tangent vector field $J H$ is a closed vector field on $\Sigma$. This means that its dual 1-form $\alpha(v)=\langle v, J H\rangle$, which is known as the Maslov form of $\phi$, is a closed form.

Therefore, if $\Sigma$ is a sphere, there exists a smooth function $f$ on $\Sigma$ such that $d f=\alpha$. As a consequence, $\alpha$ (and so $H$ ) vanish in at least two points. In particular, there are no Lagrangian (regular) immersions of two-spheres into $\mathbb{C}^{2}$ with mean curvature vector of constant length.

However, the subject is different if we consider branched immersions. In fact, we define $\Sigma_{0}$ by

$$
\Sigma_{0}=\left\{(z, w) \in \mathbb{C}^{2} \mid \Im(z \bar{w})=0,(\Re z)^{2}+(\Re w)^{2}=\left(|z|^{2}+|w|^{2}\right)^{2}\right\},
$$

where $\Re$ (respectively $\Im$ ) denotes the real part (respectively the imaginary part). Then it is an exercise to check that $\Sigma_{0}$ is a Lagrangian surface of $\mathbb{C}^{2}$ with a singularity at the origin $(0,0)$. Taking into account the embedding $\Psi: \mathbb{C}^{*} \longrightarrow \mathbb{C}^{2}$ given by

$$
\Psi(z)=\frac{2}{2|z|-i\left(|z|^{2}-1\right)}(\Re z, \Im z),
$$

we have that $\Sigma_{0}-\{(0,0)\}=\Psi\left(\mathbb{C}^{*}\right)$, and hence the surface $\Sigma_{0}-\{(0,0)\}$ is diffeomorphic to $\mathbb{C}^{*}$. The metric induced in $\mathbb{C}^{*}$ by $\Psi$ is $|d z|^{2} /\left(1+|z|^{2}\right)^{2}$, which is the

Received by the editors February 11, 2003.

2000 Mathematics Subject Classification. Primary 53C42, 53B25; Secondary 53A05, 53D12.

Key words and phrases. Constant length mean curvature vector, Lagrangian surfaces.

Research partially supported by an MCYT and FEDER grant BFM2001-2967. 

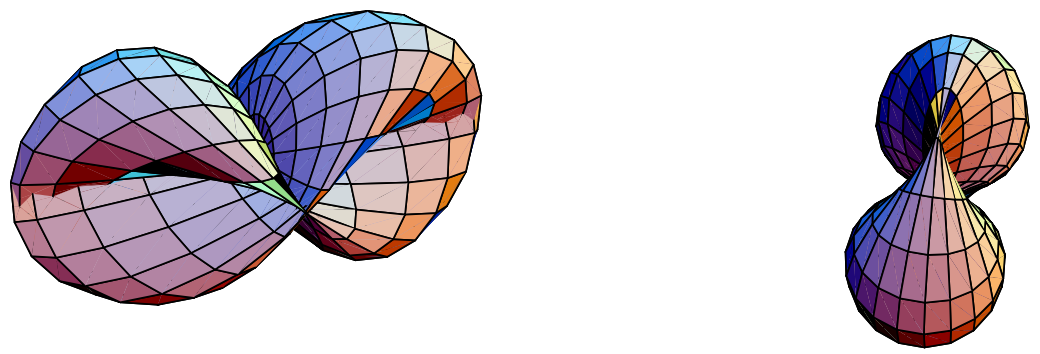

FiguRE 1. Projections on 3 -spaces in $\mathbb{C}^{2} \equiv \mathbb{R}^{4}$ of the Lagrangian pseudosphere

restriction to $\mathbb{C}^{*}$ of the canonical metric of constant curvature 1 on $\overline{\mathbb{C}}=\mathbb{C} \cup\{\infty\}$. Also, it is easy to obtain that the mean curvature $H$ of $\Psi$ is given by

$$
H(z)=3 \frac{\left(|z|^{4}-6|z|^{2}+1\right)-4 i|z|\left(|z|^{2}-1\right)}{2|z|\left(1+|z|^{2}\right)^{2}}(\Re z, \Im z),
$$

and that $H$ has constant length with $|H|^{2}=9 / 4$. If we use the branched covering of degree two of the sphere, $z \in \overline{\mathbb{C}} \mapsto z^{2} \in \overline{\mathbb{C}}$, we have a branched immersion $\Phi: \overline{\mathbb{C}} \rightarrow \mathbb{C}^{2}$,

$$
\Phi(z)=\frac{2}{2|z|^{2}-i\left(|z|^{4}-1\right)}\left(\Re\left(z^{2}\right), \Im\left(z^{2}\right)\right),
$$

whose branched points are 0 and $\infty$ and whose image is $\Sigma_{0}$. This surface is known as the Lagrangian pseudosphere (see Figure 1), and it was defined by B. Y. Chen in a different way (see Example 2.2 in [1]).

The aim of this paper is to prove the following uniqueness result.

Theorem 1. Let $\phi: \Sigma \rightarrow \mathbb{C}^{2}$ be a branched Lagrangian immersion of a sphere $\Sigma$. If the mean curvature $H$ of $\phi$ has constant length, then $\phi(\Sigma)$ is congruent, up to dilations, to the Lagrangian pseudosphere.

The main idea in the proof of this result is to construct on such a surface a holomorphic 3-differential and to make use of the Riemann-Roch Theorem. The statement of our theorem can be interpreted in the spirit of the classical Hopf's theorem characterizing the round sphere as the only genus zero oriented surface with constant mean curvature in $\mathbb{R}^{3}$.

\section{Proof of the theorem}

Throughout this section we follow [2] for the definition and general properties of branched immersions.

Let $\bar{\nabla}$ be the connection on $\phi^{*} T \mathbb{C}^{2}$ induced by the Levi-Civita connection on $\mathbb{C}^{2}$. We have the decomposition

$$
\bar{\nabla}=\nabla+\nabla^{\perp}
$$

where $\nabla$ is the Levi-Civita connection of the induced metric on $\Sigma$ by $\phi$ (which will also be denoted by $\langle\rangle$,$) and \nabla^{\perp}$ is the normal connection. The formulas of Gauss 
and Weingarten lead to

$$
\nabla_{Y}^{\perp} J Z=J \nabla_{Y} Z
$$

and so $\langle\sigma(X, Y), J Z\rangle$ is totally symmetric for any tangent vector fields $X, Y$ and $Z$. We consider a global isothermal parameter $z=x+i y$ on $\Sigma=\overline{\mathbb{C}}=\mathbb{C} \cup\{\infty\}$, in such a way that

$$
\left\langle\phi_{z}, \phi_{z}\right\rangle=0, \quad\left|\phi_{z}\right|^{2} \geq 0
$$

where $\phi_{z}$ vanishes in the isolated singularities of the branched immersion $\phi$, and the derivatives with respect to $z$ and $\bar{z}$ are taken to be

$$
\partial_{z}=\frac{1}{2}\left(\frac{\partial}{\partial x}-i \frac{\partial}{\partial y}\right), \quad \partial_{\bar{z}}=\frac{1}{2}\left(\frac{\partial}{\partial x}+i \frac{\partial}{\partial y}\right) .
$$

The Gauss equation is written as

$$
\phi_{z z}=\left(\log \left|\phi_{z}\right|^{2}\right)_{z} \phi_{z}+\sigma_{z z}, \quad \phi_{z \bar{z}}=\left|\phi_{z}\right|^{2} H,
$$

where $\sigma_{z z}=\sigma\left(\phi_{z}, \phi_{z}\right)$.

Since $\phi$ cannot be minimal because our surface is compact, we can assume that $|H|^{2}$ is nonzero. Also, up to dilations, we can take $|H|^{2}=9 / 4$.

Let $\Theta$ be the 3-differential on $\overline{\mathbb{C}}$ defined by

$$
\Theta=\left(27\left\langle\sigma_{z z}, J \phi_{z}\right\rangle+8\left\langle H, J \phi_{z}\right\rangle^{3}\right) \otimes(d z)^{3} .
$$

We are going to prove that $\Theta$ is meromorphic. We work outside the branched points. From the general properties of Lagrangian immersions mentioned above and (2.1), it follows that

$$
\left\langle\sigma_{z z}, J \phi_{z}\right\rangle_{\bar{z}}=\left\langle(\nabla \sigma)\left(\partial_{z}, \partial_{z}, \partial_{\bar{z}}\right), J \phi_{z}\right\rangle=\left|\phi_{z}\right|^{2}\left\langle H_{z}, J \phi_{z}\right\rangle
$$

Using again the Lagrangian character of $\phi$ and the fact that $J H$ is a closed vector field, we obtain

$$
\left(\left\langle H, J \phi_{z}\right\rangle^{3}\right)_{\bar{z}}=3\left\langle H, J \phi_{z}\right\rangle^{2}\left\langle H_{\bar{z}}, J \phi_{z}\right\rangle=3\left\langle H, J \phi_{z}\right\rangle^{2}\left\langle H_{z}, J \phi_{\bar{z}}\right\rangle .
$$

But

$$
0=|H|_{z}^{2}=2\left\langle H_{z}, H\right\rangle=2\left|\phi_{z}\right|^{-2}\left(\left\langle H, J \phi_{z}\right\rangle\left\langle H_{z}, J \phi_{\bar{z}}\right\rangle+\left\langle H, J \phi_{\bar{z}}\right\rangle\left\langle H_{z}, J \phi_{z}\right\rangle\right) .
$$

Thus, the above equation becomes

$$
\left(\left\langle H, J \phi_{z}\right\rangle^{3}\right)_{\bar{z}}=-\frac{27\left|\phi_{z}\right|^{2}}{8}\left\langle H_{z}, J \phi_{z}\right\rangle .
$$

Hence we obtain from (2.2), (2.3) and (2.4) that $\bar{\partial} \Theta=0$, and so $\Theta$ is meromorphic.

Looking at the expression of $\Theta$ in (2.2), the first term has zeroes at the branched points and the second one also has zeroes at the branched points because $|H|^{2}$ is a non-null constant. Therefore, $\Theta$ has no poles and it is a holomorphic 3-differential on $\overline{\mathbb{C}}$. Now the Riemann-Roch Theorem says that $\Theta$ vanishes identically, which means that

$$
27\left\langle\sigma_{z z}, J \phi_{z}\right\rangle=-8\left\langle H, J \phi_{z}\right\rangle^{3} .
$$

If $K$ is the Gauss curvature of $\Sigma$, it is easy to check that

$$
\left|\left\langle\sigma_{z z}, J \phi_{z}\right\rangle\right|^{2}=\frac{\left|\phi_{z}\right|^{6}}{8}(9-8 K), \quad\left|\left\langle H, J \phi_{z}\right\rangle\right|^{2}=\frac{9\left|\phi_{z}\right|^{2}}{8} .
$$

So $\Theta \equiv 0$ implies that $K \equiv 1$. 
On the other hand, from (2.1), (2.5) and the properties of the Lagrangian surfaces, it follows that

$$
\begin{aligned}
\sigma_{z z}= & \left|\phi_{z}\right|^{-2}\left(\left\langle\sigma_{z z}, J \phi_{\bar{z}}\right\rangle J \phi_{z}+\left\langle\sigma_{z z}, J \phi_{z}\right\rangle J \phi_{\bar{z}}\right) \\
& =\left\langle H, J \phi_{z}\right\rangle J \phi_{z}-\frac{8\left|\phi_{z}\right|^{-2}}{27}\left\langle H, J \phi_{z}\right\rangle^{3} J \phi_{\bar{z}} .
\end{aligned}
$$

Since $J H=-2\left|\phi_{z}\right|^{-2} \Re\left(\left\langle H, J \phi_{\bar{z}}\right\rangle \phi_{z}\right)$, using (2.1) and (2.6) it is not difficult to see that

$$
\sigma(J H, J H)=3 H \text {. }
$$

Since $J H$ is a closed vector field of constant length, in addition one gets that $\nabla_{J H} J H=0$. This means that $J H$ is a geodesic vector field, i.e., the integral curves of $J H$ are geodesics on $\overline{\mathbb{C}}$. This fact, joined to (2.7), says that $\bar{\nabla}_{J H} J H=3 H$. Then any integral curve $\alpha$ of $J H$ is applied by $\phi$ in a curve $\gamma=\phi(\alpha)$ in $\mathbb{C}^{2}$ satisfying $\gamma^{\prime \prime}=-3 J \gamma^{\prime}$. In particular, this shows that $\gamma$ is a circle of radius 3 in a complex plane of $\mathbb{C}^{2}$ parameterized by $\left|\gamma^{\prime}\right|=3 / 2$. Hence our immersion $\phi$ can be locally reparameterized as follows:

$$
\phi: I \times \mathbb{R} \rightarrow \mathbb{C}^{2}, \quad \phi(s, t)=c(s)+\frac{e^{-3 i t}}{2} \psi(s),
$$

where $I$ is an open interval in $\mathbb{R}$ with $0 \in I, c(s): I \rightarrow \mathbb{C}^{2}$ is the curve describing the centers of the circles, and $\psi: I \rightarrow \mathbb{S}^{3}(1) \subset \mathbb{C}^{2}$ is the curve describing the linear complex planes of the circles.

First we are going to see that, up to reparametrizations, the spherical curve $\psi$ can be chosen with the property that $\left\langle\psi^{\prime}(s), J \psi(s)\right\rangle=0, \forall s \in I$. In fact, we consider the diffeomorphism $F: I \times \mathbb{R} \rightarrow I \times \mathbb{R}$ given by

$$
F(s, t)=\left(s, t+\frac{1}{3} \int_{0}^{s}\left\langle\psi^{\prime}(r), J \psi(r)\right\rangle d r\right) .
$$

Then the new immersion $\tilde{\phi}(s, t)=\phi(F(s, t))$ satisfies $\left\langle\tilde{\psi}^{\prime}(s), J \tilde{\psi}(s)\right\rangle=0, \forall(s, t) \in$ $I \times \mathbb{R}$. Renaming $\tilde{\psi}$ by $\psi$, we can assume that $\left\langle\psi^{\prime}, J \psi\right\rangle=0$.

With this parameterization, $t \mapsto \phi\left(s_{0}, t\right)$ is an integral curve of the vector field $J H$, and so

$$
H(s, t)=-J \phi_{t}(s, t), \quad \forall(s, t) \in I \times \mathbb{R} .
$$

In order to make use of (2.8), we proceed as follows. If $\psi=\left(\psi_{1}, \psi_{2}\right)$, we set $\hat{\psi}=\left(-\bar{\psi}_{2}, \bar{\psi}_{1}\right)$. In this way, $\{\psi, \hat{\psi}\}$ will be an orthonormal complex reference in $\mathbb{C}^{2}$. The following equations will be useful:

$$
\psi^{\prime}=h \hat{\psi}, \quad \hat{\psi}^{\prime}=-\bar{h} \psi,
$$

where $h: I \rightarrow \mathbb{C}$ is a complex function. Since $\phi$ is a Lagrangian immersion, we have that $\left\langle\phi_{t}, J \phi_{s}\right\rangle=0$. This means that $\Re\left(e^{3 i t}\left(\left\langle c^{\prime}(s), \psi(s)\right\rangle+i\left\langle c^{\prime}(s), J \psi(s)\right\rangle\right)=\right.$ $0, \forall(s, t) \in I \times \mathbb{R}$. Since this is true for any $t \in \mathbb{R}$, we obtain that $\left\langle c^{\prime}(s), \psi(s)\right\rangle=$ $\left\langle c^{\prime}(s), J \psi(s)\right\rangle=0 \forall s \in I$. This says that

$$
c^{\prime}(s)=a(s) \hat{\psi}(s), \quad \forall s \in I,
$$

where $a: I \rightarrow \mathbb{C}$ is a complex function. Using (2.9) and (2.10), we get

$$
e_{1}:=\phi_{s}=\left(a+\frac{e^{-3 i t}}{2} h\right) \hat{\psi}, \quad e_{2}:=\phi_{t}=-\frac{3 i}{2} e^{-3 i t} \psi
$$


and then the induced metric is given by

$$
g_{11}=|a|^{2}+\frac{|h|^{2}}{4}+\Re\left(e^{-3 i t} h \bar{a}\right), \quad g_{12}=0, \quad g_{22}=\frac{9}{4},
$$

which implies that

$$
\operatorname{det}\left(g_{i j}\right)=\frac{9}{4}\left(|a|^{2}+\frac{|h|^{2}}{4}+\Re\left(e^{-3 i t} h \bar{a}\right)\right) .
$$

Now we are going to compute the mean curvature $H$ of $\phi$. Since $2 H=\sum_{i, j=1}^{2} g^{i j} \sigma_{i j}$, where $\left(g^{i j}\right)$ is the inverse matrix of $\left(g_{i j}\right)$ and $\sigma_{i j}=\sigma\left(e_{i}, e_{j}\right)$, using (2.8) we have that

$$
\begin{aligned}
-9 / 2 & =\left(1 / g_{11}\right)\left\langle\sigma_{11}, J e_{2}\right\rangle+(4 / 9)\left\langle\sigma_{22}, J e_{2}\right\rangle, \\
0 & =\left(1 / g_{11}\right)\left\langle\sigma_{11}, J e_{1}\right\rangle+(4 / 9)\left\langle\sigma_{22}, J e_{1}\right\rangle .
\end{aligned}
$$

Taking derivatives in (2.11), after a long but straightforward computation, we conclude that the above equations simply mean that

$$
|a|^{2}=\frac{|h|^{2}}{4}, \quad \Im\left(a^{\prime} \bar{a}+\frac{h^{\prime} \bar{h}}{4}\right)=0 .
$$

Integrating this equation, we obtain

$$
a(s)=e^{i \theta} \frac{\bar{h}(s)}{2}, \quad \theta \in \mathbb{R} .
$$

Thus (2.12) and (2.13) say that

$$
\operatorname{det}\left(g_{i j}(s, t)\right)=\frac{9}{8}\left(|h|^{2}(s)+\Re\left(h^{2}(s) e^{-i(\theta+3 t)}\right)\right) .
$$

If $h\left(s_{0}\right)=0$ for some $s_{0} \in I$, then $\operatorname{det}\left(g_{i j}\left(s_{0}, t\right)\right)=0$ for all $t \in \mathbb{R}$. Hence the circle $\left\{\phi\left(s_{0}, t\right), t \in \mathbb{R}\right\}$ is singular in our surface. This is impossible because the singular points are isolated. So $\left|\psi^{\prime}(s)\right|^{2}=|h|^{2}(s)>0, \forall s \in I$. By considering the Hopf fibration $\Pi: \mathbb{S}^{3}(1) \rightarrow \mathbb{S}^{2}(1 / 2)$ onto the 2-sphere of radius $1 / 2$, denoting $\beta(s)=\Pi \circ \psi(s)$, we obtain that $\left|\beta^{\prime}(s)\right|^{2}=\left|\psi^{\prime}(s)\right|^{2}>0, \forall s \in I$. This means that the curve $\beta$ is regular, and so we can reparameterize our immersion in order to get $\left|\beta^{\prime}\right|^{2}=1$. We remark that $\psi$ is a horizontal lift of $\beta$ with respect to $\Pi$, which is unique up to rotations in $\mathbb{S}^{3}(1)$.

On the other hand, taking derivatives in (2.9), we obtain that $\Im\left(h^{\prime} \bar{h}\right)=\left\langle\psi^{\prime \prime}, J \psi^{\prime}\right\rangle$. Since $|h|^{2}=\left|\beta^{\prime}\right|^{2}=1$, if $h(s)=e^{i \alpha(s)}$, we have that $\Im\left(h^{\prime} \bar{h}\right)=\alpha^{\prime}$, and so $\alpha^{\prime}=\left\langle\psi^{\prime \prime}, J \psi^{\prime}\right\rangle$. Analyzing the Hopf fibration $\Pi$, it is not difficult to check that the curvature $k$ of the curve $\beta$ is given by $k(s)=\left\langle\psi^{\prime \prime}(s), J \psi^{\prime}(s)\right\rangle$, which implies that

$$
h(s)=e^{i \int_{0}^{s} k(r) d r}, \quad \forall s \in I .
$$

Finally, from (2.14) and (2.15), we deduce that

$$
\operatorname{det}\left(g_{i j}(s, t)\right)=\frac{9}{8}\left(1+\Re\left(e^{-i\left(\theta+3 t-2 \int_{0}^{s} k(r) d r\right)}\right)\right) .
$$

Therefore the set of singularities of $\phi$ is

$$
\left\{(s, t) \in I \times \mathbb{R} \mid 3 t=-\theta+2 \int_{0}^{s} k(r) d r+(2 n+1) \pi, n \in \mathbb{Z}\right\} .
$$


Since $\phi$ is $(2 \pi / 3)$-periodic in $t$, we can restrict $\phi$ to the set

$$
\left\{(s, t) \in I \times \mathbb{R} \mid-\theta+2 \int_{0}^{s} k(r) d r-\pi \leq 3 t \leq-\theta+2 \int_{0}^{s} k(r) d r+\pi\right\} .
$$

Its boundary is applied by $\phi$ in the curve $\eta(s)=c(s)-\frac{1}{2} e^{i\left(\theta-2 \int_{0}^{s} k(r) d r\right)} \psi(s)$. This singular curve must be a point, because our immersion from a sphere in $\mathbb{C}^{2}$ has only isolated singular points. Using (2.9), (2.10), (2.13) and (2.15), we see that

$$
0=\eta^{\prime}(s)=i k(s) e^{i\left(\theta-2 \int_{0}^{s} k(r) d r\right)} \psi(s),
$$

which shows that $k \equiv 0$. Hence $\beta$ must be a great circle in $\mathbb{S}^{2}(1 / 2)$. It can be parameterized by $\beta(s)=\frac{1}{2}(\cos 2 s, \sin 2 s, 0), s \in \mathbb{R}$. In this case, we conclude that $h=1, a=e^{i \theta} / 2$, and so (2.9) and (2.10) imply that $c=c_{0}+\left(e^{i \theta} / 2\right) \psi, c_{0} \in \mathbb{C}^{2}$. Since $\psi$ is a horizontal lift of $\beta$, we can take $\psi(s)=\frac{e^{i \theta}}{\sqrt{2}}\left(e^{i s}, e^{-i s}\right)$. Therefore our immersion $\phi$ can be written as

$$
\phi(s, t)=c_{0}+\frac{e^{i \theta}\left(1+e^{-3 i t}\right)}{2 \sqrt{2}}\left(e^{i s}, e^{-i s}\right), \quad(s, t) \in \mathbb{R}^{2} .
$$

Up to a translation and the holomorphic isometry of $\mathbb{C}^{2}$ given by the unitary matrix

$$
\frac{e^{-i \theta}}{\sqrt{2}}\left(\begin{array}{c|c}
1 & -i \\
\hline 1 & i
\end{array}\right)
$$

it is clear that our immersion is rewritten as

$$
\phi(s, t)=\frac{1+e^{-3 i t}}{2}(\cos s, \sin s), \quad(s, t) \in \mathbb{R}^{2} .
$$

Then $\phi$ is $2 \pi$-periodic in $s$, and so it defines an immersion from $\mathbb{S}^{1} \times \mathbb{R}$ in $\mathbb{C}^{2}$, such that the set of singular points $\left\{\mathbb{S}^{1} \times\left\{\frac{2 n+1}{3} \pi\right\}, n \in \mathbb{Z}\right\}$ is applied by $\phi$ in $(0,0) \in \mathbb{C}^{2}$. Using again that $\phi$ is $(2 \pi / 3)$-periodic in $t$, if in $\mathbb{S}^{1} \times[-\pi / 3, \pi / 3]$ we identify $\mathbb{S}^{1} \times\{-\pi / 3\}$ and $\mathbb{S}^{1} \times\{\pi / 3\}$ to two different points, we obtain an immersion from a sphere to $\mathbb{C}^{2}$, which is the Lagrangian pseudosphere.

\section{FinAL REMARKS}

In the proof of the theorem we have also classified locally the Lagrangian surfaces of $\mathbb{C}^{2}$ with non-null constant length mean curvature vector such that the 3 -differential $\Theta$ defined in (2.2) vanishes identically. We emphasize that they can be described in terms of curves of $\mathbb{S}^{2}(1 / 2)$. In fact, we can summarize the above reasoning in the following result:

Let $\phi: \Sigma \rightarrow \mathbb{C}^{2}$ be a non-null constant length mean curvature vector Lagrangian immersion with $\Theta \equiv 0$. Then $\phi$ is locally congruent to

$$
(s, t) \in I \times \mathbb{R} \longmapsto c(s)+\frac{e^{-3 i t}}{2} \psi(s) \in \mathbb{C}^{2},
$$

where $\psi: I \rightarrow \mathbb{S}^{3}(1)$ is a horizontal lift with respect to the Hopf fibration $\Pi: \mathbb{S}^{3}(1) \rightarrow \mathbb{S}^{2}(1 / 2)$ of a unit speed curve $\beta$, and $c$ is the curve that is solution of the o.d.e.

$$
c^{\prime}(s)=\frac{1}{2} e^{-2 i \int_{0}^{s} k(r) d r} \psi^{\prime}(s),
$$

where $k$ is the curvature of $\beta$. 


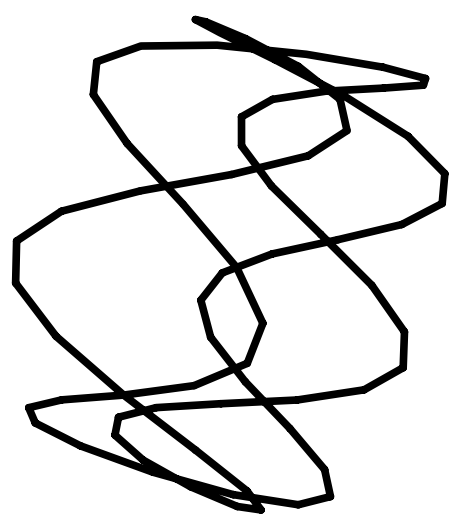

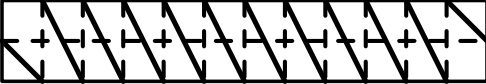

Figure 2. The curve of singular points of the torus: a projection on a 3 -space in $\mathbb{C}^{2} \equiv \mathbb{R}^{4}$ of its image by $\varphi$ and its representation in the lattice $\Lambda$

The Lagrangian surface corresponding to the curve $\beta$ with zero curvature is the Lagrangian pseudosphere. Besides this, the easiest curves in $\mathbb{S}^{2}(1 / 2)$ are those of constant curvature.

To illustrate with an example, we consider the unit speed curve with constant curvature $-3 / 2$ in $\mathbb{S}^{2}(1 / 2)$ given by

$$
\beta(s)=\frac{1}{10}(4 \cos (5 s / 2), 4 \sin (5 s / 2), 3) .
$$

It is an exercise to check that its associated Lagrangian immersion (according to the above result) can be written, up to dilations and congruences, as follows:

$$
\varphi(s, t)=\left(e^{-i(3 t-s / 2)}+\frac{1}{7} e^{7 i s / 2}, \frac{1}{2} e^{-i(3 t+2 s)}-e^{i s}\right), \quad(t, s) \in \mathbb{R}^{2} .
$$

It is clear that $\varphi$ is doubly periodic with respect to the lattice $\Lambda=\operatorname{span}\{(4 \pi, 0)$, $(0,2 \pi / 3)\}$. Hence $\varphi=\varphi(s, t),(s, t) \in \Lambda$, provides a Lagrangian immersion of a torus with non-null constant length mean curvature vector and positive constant curvature in $\mathbb{C}^{2}$, whose set of singular points is given by the curve

$$
\{(t, s) \mid t+s= \pm \pi / 3\},
$$

which is applied by $\varphi$ in a fully curve in $\mathbb{C}^{2}$ (see Figure 2 ).

\section{REFERENCES}

1. B. Y. Chen, Complex extensors and Lagrangian submanifolds in complex Euclidean spaces, Tôhoku Math. J. 49 (1997), 277-297. MR 98g:53096 
2. R. Gulliver, R. Osserman and H. Royden, A theory of branched immersions of surfaces, Amer. J. Math. 95 (1973), 750-812. MR 50:14595]

Departamento de Matemáticas, Escuela Politécnica Superior, Universidad de Jaén, 23071 JAÉn, SPAIN

E-mail address: icastro@ujaen.es

Departamento de Geometría y Topología, Universidad de Granada, 18071 Granada, SPAIN

E-mail address: furbano@ugr.es 